Case Report

\title{
Uterine Carcinosarcoma in a 2-year-old Female Wistar Hannover GALAS Rat
}

\author{
Shigeaki Takami1^,\#, Kumiko Ogawa1 ${ }^{\text {, Takashi Umemura }}{ }^{1}$, Daisuke Hibi1, Yuji Ishii ${ }^{1}$, \\ Toshiya Okamura1, Masako Tasaki¹, Tomoki Inoue1, Yuta Suzuki¹, Meilan Jin¹, \\ Young-Man $\mathrm{Cho}^{1}$, and Akiyoshi Nishikawa ${ }^{1}$ \\ ${ }^{1}$ Division of Pathology, National Institute of Health Sciences, 1-18-1 Kamiyoga, Setagaya-ku, Tokyo 158-8501, Japan \\ \# Present: Pathology and Clinical Examination Laboratory, Safety Assessment Unit, Biosafety Research Center, Foods, \\ Drugs and Pesticides, 582-2 Shioshinden, Iwata, Shizuoka 437-1213, Japan
}

\begin{abstract}
Carcinosarcomas are rare tumors in humans as well as rats and most commonly occur in the uterus. Recently, we observed a case of incidental carcinosarcoma of the uterus in a female Wistar Hannover GALAS [BrlHan:WIST@ Jcl (GALAS)] rat at 2 years of age. Histopathologically, the tumor was characterized by an admixture of malignant epithelial and nonepithelial elements. The carcinomatous components represented a type of endometrial carcinoma, consisting of glandular and solid proliferation of large-sized tumor cells. Prominent mitoses and tumor cell invasion were observed. The sarcomatous components were characterized by multifocal proliferation of severe atypical cells with cartilage matrix and were diagnosed as chondrosarcoma. Transitions between carcinomatous and sarcomatous components were observed, and many tumor cells in the solid lesion showed immunohistochemical reactivity with both cytokeratin and vimentin. Based on these findings, this tumor was diagnosed as a uterine carcinosarcoma. This is the first report of uterine carcinosarcoma in Wistar Hannover GALAS [BrlHan:WIST@Jcl (GALAS)] rats. (DOI: 10.1293/tox.24.63; J Toxicol Pathol 2011; 24: 63-67)
\end{abstract}

Key words: carcinosarcoma, uterus, spontaneous tumor, Wistar Hannover GALAS rat

Carcinosarcomas are rare tumors that most commonly develop in the female genital tract, particularly in the uterus ${ }^{1,2}$. Characteristic histopathological findings are coexistence of both epithelial and nonepithelial malignant components 1,2 . Development of carcinosarcomas has been demonstrated in various human organs, including the uterus $^{1,2}$, ovary ${ }^{3,4}$, breast $^{5}$, lung ${ }^{6,7}$ and gastrointestinal tract ${ }^{8-11}$. Although spontaneous carcinosarcomas in the uterus of LEWIS rats ${ }^{12}$ and mammary gland of SD rats ${ }^{13}$ have been documented, no reports about this tumor type in Wistar Hannover GALAS rats have been published. In this article, we report a case of carcinosarcoma of the uterus in a female Wistar Hannover GALAS rat at 2 years of age.

In this study, Wistar Hannover GALAS [BrlHan: WIST@Jcl (GALAS)] rats (CLEA Japan, Tokyo, Japan)

(C)2011 The Japanese Society of Toxicologic Pathology

Received: 18 August 2010, Accepted: 12 October 2010

Mailing address: Shigeaki Takami, Pathology and Clinical Examination Laboratory, Safety Assessment Unit, Biosafety Research Center, Foods, Drugs and Pesticides, 582-2 Shioshinden, Iwata, Shizuoka 437-1213, Japan

TEL: 81-538-58-1266 FAX: 81-538-58-3586

E-mail: takami-shigeaki@anpyo.or.jp were randomly allocated to three groups, each consisting of 50 males and 50 females, and groups of rats of each sex were fed a tocotrienol-containing basal diet (CE-2; CLEA Japan, Tokyo, Japan) and tap water ad libitum for 104 weeks beginning at 6 weeks of age. The tocotrienol dose levels in the diet in the high-dose group were $2.0 \%$ during weeks $1-50$ and $1.0 \%$ for the remainder of the study. The tocotrienol (Eisai, Tokyo, Japan) given in this study was composed of $\alpha$-tocotrienol (21.4\%), $\beta$-tocotrienol (3.5\%), $\gamma$-tocotrienol $(36.5 \%), \delta$-tocotrienol $(8.6 \%), \alpha$-tocopherol $(20.5 \%), \beta$ tocopherol $(0.7 \%), \gamma$-tocopherol $(1.0 \%)$ and $\delta$-tocopherol $(0.5 \%)$. The animals were housed in a clear polycarbonate cage with sterilized white wood chips (Sankyo Labo Service Corporation, Tokyo, Japan) for bedding in a standard air-conditioned room $\left(24 \pm 1{ }^{\circ} \mathrm{C}, 55 \pm 5 \%\right.$ relative humidity, $12 \mathrm{~h} \mathrm{light/dark} \mathrm{cycles).} \mathrm{At} \mathrm{the} \mathrm{end} \mathrm{of} \mathrm{experiment,} \mathrm{we} \mathrm{found}$ one animal with a large mass $(1.0 \times 0.9 \times 0.8 \mathrm{~cm})$ in the left horn of the uterus within the 47 animals in the high-dose group. The mass was dissected with the uterus and fixed in $10 \%$ neutral buffered formalin. Macroscopically, nodules were also observed in the pituitary gland and pectoral subcutis. After fixation, the tissues were routinely processed, i.e., embedded in paraffin, sectioned at $4 \mu \mathrm{m}$ and stained with hematoxylin and eosin (HE). Additionally, toluidine 


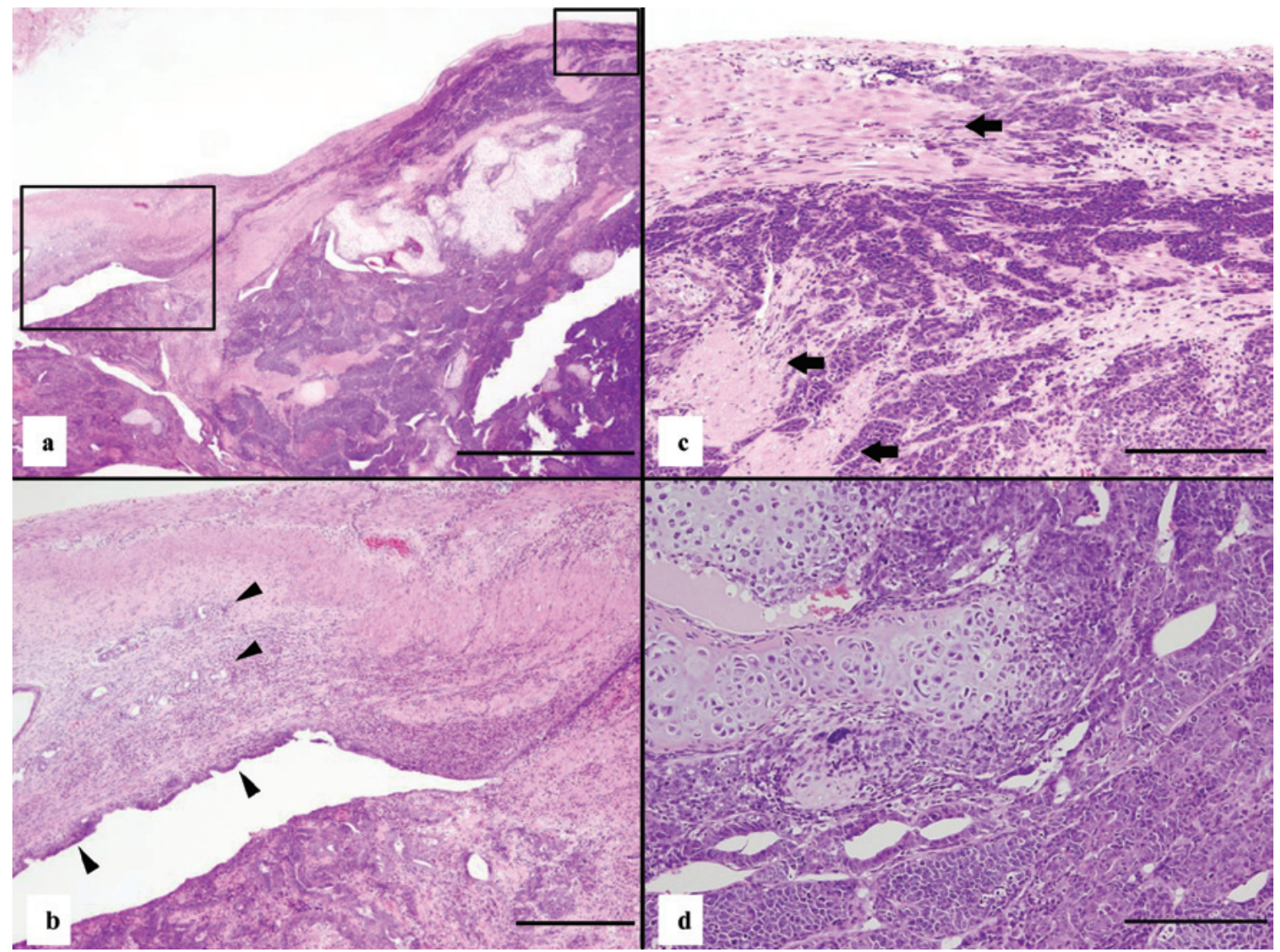

Fig. 1. Uterine carcinosarcoma. (a) Overall picture of the uterine carcinosarcoma. HE stain. Bar=2 mm. (b) High magnification of the region enclosed in the large square in a. The upper half is an existing uterine area. The arrowheads indicate existing endometrium and uterine glands. The lower half is the tumor area. HE stain. Bar=400 $\mu \mathrm{m}$. (c) High magnification of the region enclosed in the small square in a. Invasion of tumor cells is observed from the myometrium into the serosa. The arrows indicate the myometrium. HE stain. Bar=200 $\mu \mathrm{m}$. (d) Epithelial and nonepithelial malignant components are observed in a nodule. HE stain. Bar $=200 \mu \mathrm{m}$.

blue stain and immunohistochemical staining for rabbit anti-cow S-100 protein (DAKO, Glostrup, Denmark), antihuman cytokeratin (clones AE1/AE3; DAKO), anti-porcine vimentin (clone V9; DAKO) and anti-human E-cadherin (clone 36; BD Biosciences, Franklin Lakes, NJ, USA) were performed using serial sections. The animal experimental protocol was reviewed and approved by the Animal Care and Use Committee of the National Institute of Health Sciences, Japan.

Histopathologically, a neoplastic nodule appeared to protrude into the lumen of the uterus (Fig 1a, b). Invasion of tumor cells from the myometrium into the serosa were observed (Fig 1c). Two distinct types of tumor cells were observed in this nodule (Fig 1d). The first component was characterized by a proliferative pattern of tumor cells in irregular gland-like, solid sheets or nests. This type of tumor cell had large round or elliptical nuclei with a few apparent nucleoli and scant or moderate and eosinophilic or basophilic cytoplasm (Fig 2a-c), indicating endometrial carcinoma. Severe necrosis, mineralization and multiple mitoses were observed. Tumor cells with abundant collagen invaded from the myometrium into the serosa and were either single cells or clusters of a few cells (Fig 2d). Immunohistochemically, the tumor cells showing a glandular pattern were strongly positive for cytokeratin and E-cadherin and negative for vimentin, while those with a solid pattern were weakly positive for cytokeratin, partially positive for E-cadherin and mostly positive for vimentin (Fig 2e, f). The second component, which was characterized by a proliferation of polymorphic tumor cells with a pale basophilic matrix, was focally observed within the carcinomatous component. This type of tumor cell had round or oval nuclei with severe atypia and an appreciable amount of clear to eosinophilic cytoplasm (Fig 3a, b). The pale basophilic matrix exhibited metachromasia by toluidine blue staining, indicating cartilage matrix. These tumor cells were negative for E-cadherin, weakly positive for cytokeratin and intensely positive for vimentin and S-100 protein (Fig 3c, d). Thus, this sarcomatous component was diagnosed as chondrosarcoma. Transitions between carcinomatous and sarcomatous components were 


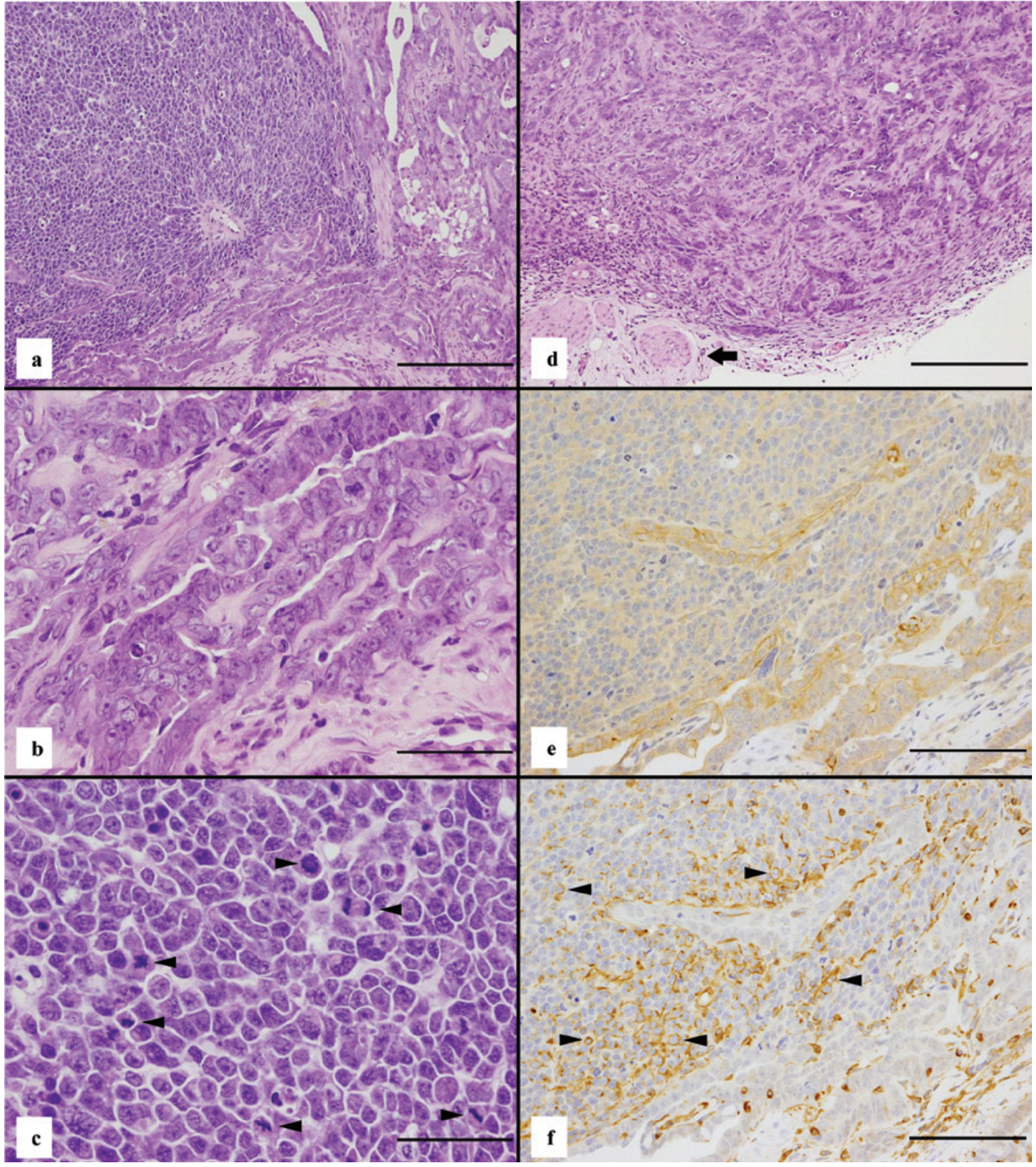

Fig. 2. Histopathological and immunohistochemical findings of carcinomatous elements in the uterine carcinosarcoma. (a) Irregular glandular structures and solid sheet structures of tumor cells. HE stain. Bar $=200 \mu \mathrm{m}$. (b) High magnification of glandular area in a. Tumor cells have large round or elliptical nuclei and moderate eosinophilic cytoplasm. HE stain. Bar $=50 \mu \mathrm{m}$. (c) High magnification of the solid sheet area in a. Tumor cells have large round or oval nuclei and scant basophilic cytoplasm. Mitotic figures are observed (arrowhead). HE stain. Bar $=50 \mu \mathrm{m}$. (d) Invasion of tumor cells with collagen is observed from the myometrium into the serosa. Many small glandular structures are seen within the tumor cells. The arrow indicates the myometrium. HE stain. Bar $=200 \mu \mathrm{m}$. (e) Immunoreactivity for cytokeratin. Glandular tumor cells are positive, while solid sheet tumor cells are weakly positive. Bar=100 $\mu \mathrm{m}$. (f) Immunoreactivity for vimentin. Glandular tumor cells are negative, while many of the tumor cells in the solid sheet area are positive (arrowhead). Bar=100 $\mu \mathrm{m}$.

observed (Fig 1a, d). Although myometrial and vascular invasion and serosal involvement were observed, no metastasis was seen. Primary carcinomas were also seen in the pituitary gland and mammary gland of this animal. Macro- scopically and microscopically, carcinosarcomas were not observed in other animals including the tocotrienol-treated and control animals.

Based on the present histopathological and immu- 


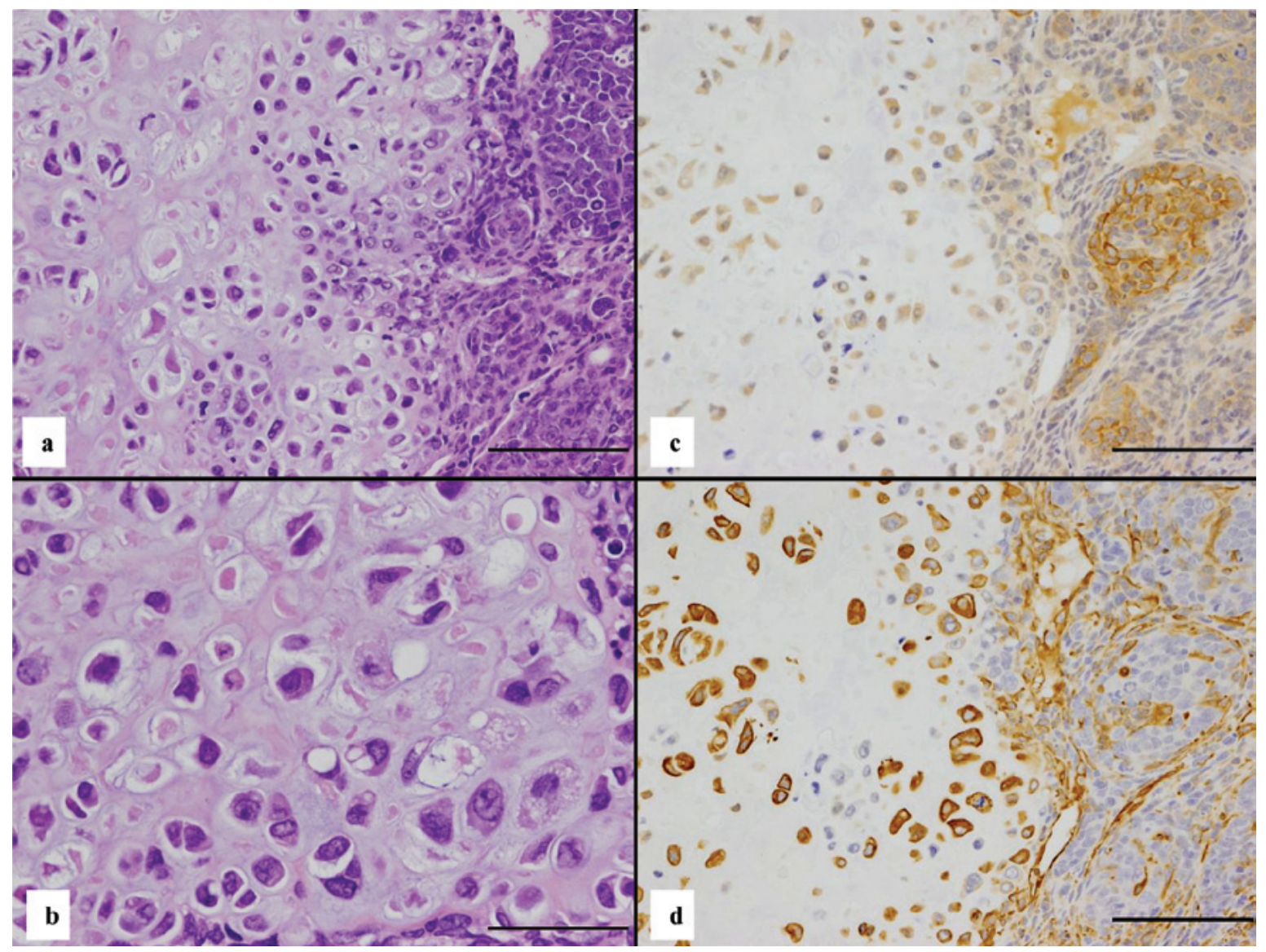

Fig. 3. Histopathological and immunohistochemical findings of sarcomatous elements in the uterine carcinosarcoma. (a) Proliferation of tumor cells with a basophilic matrix. HE stain. Bar=100 $\mu \mathrm{m}$. (b) High magnification of a. Tumor cells have round or oval nuclei and an appreciable amount of eosinophilic cytoplasm. HE stain. Bar=50 $\mu \mathrm{m}$. (c) Immunoreactivity for cytokeratin. Tumor cells of epithelial origin are positive, while those of sarcomatous components are weakly positive. Bar $=100 \mu \mathrm{m}$. (d) Immunoreactivity for vimentin. Tumor cells of epithelial origin are negative, while those of sarcomatous components are intensely positive. Bar $=100 \mu \mathrm{m}$.

nohistochemical findings, this tumor was diagnosed as a uterine carcinosarcoma. The conclusive histopathological findings supporting this diagnosis were the mixture of carcinomatous and sarcomatous elements in a single neoplastic nodule. This case was further subclassified as the heterologous type because the sarcomatous components showed typical chondrosarcoma that had developed from an element that did not originally exist. Although carcinosarcomas are unusual tumors, they are the most common type of mixed epithelial-nonepithelial endometrial tumors ${ }^{1}$. The prognosis of carcinosarcoma is generally poor due to recurrence, lymph node metastasis and myometrial and lymph-vascular space invasion ${ }^{14,15}$. Although metastasis to pelvic or periaortic lymph nodes has been reported ${ }^{1,15}$, metastasis of tumor cells was not observed in the mesenteric lymph nodes or other organs such as the lung, liver and spleen in the present case. Immunohistochemical staining for cytokeratin, a general epithelial marker, and vimentin, a typical mesenchymal marker, have suggested distinct carcinomatous and sarcomatous populations in carcinosarcomas $8,16,17$. The present immunohistochemical findings were consistent with those of the previous reports ${ }^{16,17}$. Epithelial markers are frequently expressed by sarcomatous-appearing cells, and epithelial and mesenchymal markers are coexpressed in many tumor cells in carcinosarcomas ${ }^{16,17}$. Carcinosarcomas have been suggested to result from neoplastic transformation of cells with a capacity for both epithelial and mesenchymal differentiation. The histogenesis of carcinosarcomas has been hypothesized to involve a collision tumor, combination/conversion tumor or other compositional tumorit,18-21. The presence of many carcinosarcomas has been reported to represent a combination tumor ${ }^{20,21}$. Although we did not perform a stem cell analysis, the present case may be considered to be a combination tumor based on the results of immunohistochemistry and the evidence of mixed and/or transitional pattern of carcinomatous and sarcomatous components.

Several nomenclature systems are used to describe this type of neoplastic lesion. According to the WHO classification $^{22}$, malignant mesodermal mixed tumor is synonymous with carcinosarcoma, but tumors with heterologous and 
homologous sarcomatous components are sometimes diagnosed as malignant mesodermal mixed tumor and carcinosarcoma, respectively.

The tumor in the present case was detected in only a single rat within a group of similarly treated animals. Therefore, this tumor could be incidental and may not be related to the treatment. This is the first report of uterine carcinosarcoma in a Wistar Hannover GALAS rat.

Acknowledgments: This study was supported in part by a Grant-in-Aid from the Ministry of Health, Labor and Welfare of Japan. The authors thank Ms. Ayako Kaneko and Ms. Toshimi Komatsu for skillful technical assistance.

\section{References}

1. Silverberg SG, and Kurman RJ. Mixed epithelial-nonepithelial tumors. In: Atras of Tumor Pathology, Third Series: Tumors of the Uterine Corpus and Gestational Trophoblastic Disease. J Rosai, and LH Sobin (eds). Armed Forces Institute of Pathology, Washington, D.C. 153-179. 1991.

2. Kempson RL, and Hendrickson MR. Smooth muscle, endometrial stromal, and mixed Müllerian tumors of the uterus. Mod Pathol. 13: 328-342. 2000.[Medline] [CrossRef]

3. Brown E, Stewart M, Rye T, Al-Nafussi A, Williams AR, Bradburn M, Smyth J, and Gabra H. Carcinosarcoma of the ovary: 19 years of prospective data from a single center. Cancer. 100: 2148-2153. 2004.[Medline] [CrossRef]

4. Harris MA, Delap LM, Sengupta PS, Wilkinson PM, Welch RS, Swindell R, Shanks JH, Wilson G, Slade RJ, Reynolds $\mathrm{K}$, and Jayson GC. Carcinosarcoma of the ovary. Br J Cancer. 88: 654-657. 2003.[Medline] [CrossRef]

5. Hennessy BT, Giordano S, Broglio K, Duan Z, Trent J, Buchholz TA, Babiera G, Hortobagyi GN, and Valero V. Biphasic metaplastic sarcomatoid carcinoma of the breast. Ann Oncol. 17: 605-613. 2006.[Medline] [CrossRef]

6. Sanyal K, and Sabanathan K. Lung carcinosarcoma as a rare biphasic sarcomatoid carcinoma: a case report. Cases J. 2: 7968. 2009.[Medline] [CrossRef]

7. Petrov DB, Vlassov VI, Kalaydjiev GT, Plochev MA, Obretenov ED, Stanoev VI, and Danon SE. Primary pulmonary sarcomas and carcinosarcomas-postoperative results and comparative survival analysis. Eur J Cardiothorac Surg. 23: 461-466. 2003.[Medline] [CrossRef]

8. Ota S, Kato A, Kobayashi H, Yonezumi M, Yamaguchi J, Musashi M, Imamura M, and Asaka M. Monoclonal origin of an esophageal carcinosarcoma producing granulocytecolony stimulating factor: a case report. Cancer. 82: 21022111. 1998.[Medline] [CrossRef]

9. Randjelovic T, Filipovic B, Babic D, and Cemerikic V. Carcinosarcoma of the stomach: a case report and review of the literature. World J Gastroenterol. 13: 5533-5536. 2007.[Medline]

10. Ambrosini-Spaltro A, Vaira V, Braidotti P, Rovati MP, Ferrero $\mathrm{S}$, and Bosari S. Carcinosarcoma of the colon: report of a case with morphological, ultrastructural and molecular analysis. BMC Cancer. 6: 185. 2006.[Medline] [CrossRef]

11. Tsekouras DK, Katsaragakis S, Theodorou D, Kafiri G, Archontovasilis F, Giannopoulos P, Drimousis P, and Bramis J. Rectal carcinosarcoma: a case report and review of literature. World J Gastroenterol. 12: 1481-1484. 2006.[Medline]

12. Kaspareit-Rittinghausen J, and Deerberg F. Spontaneous malignant mixed müllerian tumors and rhabdomyosarcoma of the uterus in rats. Toxicol Pathol. 18: 417-422. 1990.[Medline] [CrossRef]

13. Komitowski D, Sass B, and Laub W. Rat mammary tumor classification: notes on comparative aspects. J Natl Cancer Inst. 68: 147-156. 1982.[Medline]

14. Snyder MJ, Robboy SJ, Vollmer RT, and Dodd LG. An abnormal cervicovaginal cytology smear in uterine carcinosarcoma is an adverse prognostic sign: analysis of 25 cases. Am J Clin Pathol. 122: 434-439. 2004.[Medline] [CrossRef]

15. Yamada SD, Burger RA, Brewster WR, Anton D, Kohler MF, and Monk BJ. Pathologic variables and adjuvant therapy as predictors of recurrence and survival for patients with surgically evaluated carcinosarcoma of the uterus. Cancer. 88: 2782-2786. 2000.[Medline] [CrossRef]

16. de Brito PA, Silverberg SG, and Orenstein JM. Carcinosarcoma (malignant mixed müllerian (mesodermal) tumor) of the female genital tract: immunohistochemical and ultrastructural analysis of 28 cases. Hum Pathol. 24: 132-142. 1993.[Medline] [CrossRef]

17. Bitterman P, Chun B, and Kurman RJ. The significance of epithelial differentiation in mixed mesodermal tumors of the uterus. A clinicopathologic and immunohistochemical study. Am J Surg Pathol. 14: 317-328. 1990.[Medline] [CrossRef]

18. McCluggage WG. Malignant biphasic uterine tumours: carcinosarcomas or metaplastic carcinomas? J Clin Pathol. 55: 321-325. 2002.[Medline]

19. Gorai I, Yanagibashi T, Taki A, Udagawa K, Miyagi E, Nakazawa T, Hirahara F, Nagashima Y, and Minaguchi H. Uterine carcinosarcoma is derived from a single stem cell: an in vitro study. Int J Cancer. 72: 821-827. 1997.[Medline] [CrossRef]

20. Fujii H, Yoshida M, Gong ZX, Matsumoto T, Hamano Y, Fukunaga M, Hruban RH, Gabrielson E, and Shirai T. Frequent genetic heterogeneity in the clonal evolution of gynecological carcinosarcoma and its influence on phenotypic diversity. Cancer Res. 60: 114-120. 2000.[Medline]

21. Wada H, Enomoto T, Fujita M, Yoshino K, Nakashima R, Kurachi H, Haba T, Wakasa K, Shroyer KR, Tsujimoto M, Hongyo T, Nomura T, and Murata Y. Molecular evidence that most but not all carcinosarcomas of the uterus are combination tumors. Cancer Res. 57: 5379-5385. 1997.[Medline]

22. McCluggage WG, Haller U, Kurman RJ, and Kubik-Huch RA. Mixed epithelial and mesenchymal tumours. In: World Health Organization Classification of Tumours: Pathology and Genetics of Tumours of the Breast and Female Genital Organs. FA Tavassoli, and P Devilee (eds). IARC Press, Lyon. 245-249. 2003. 